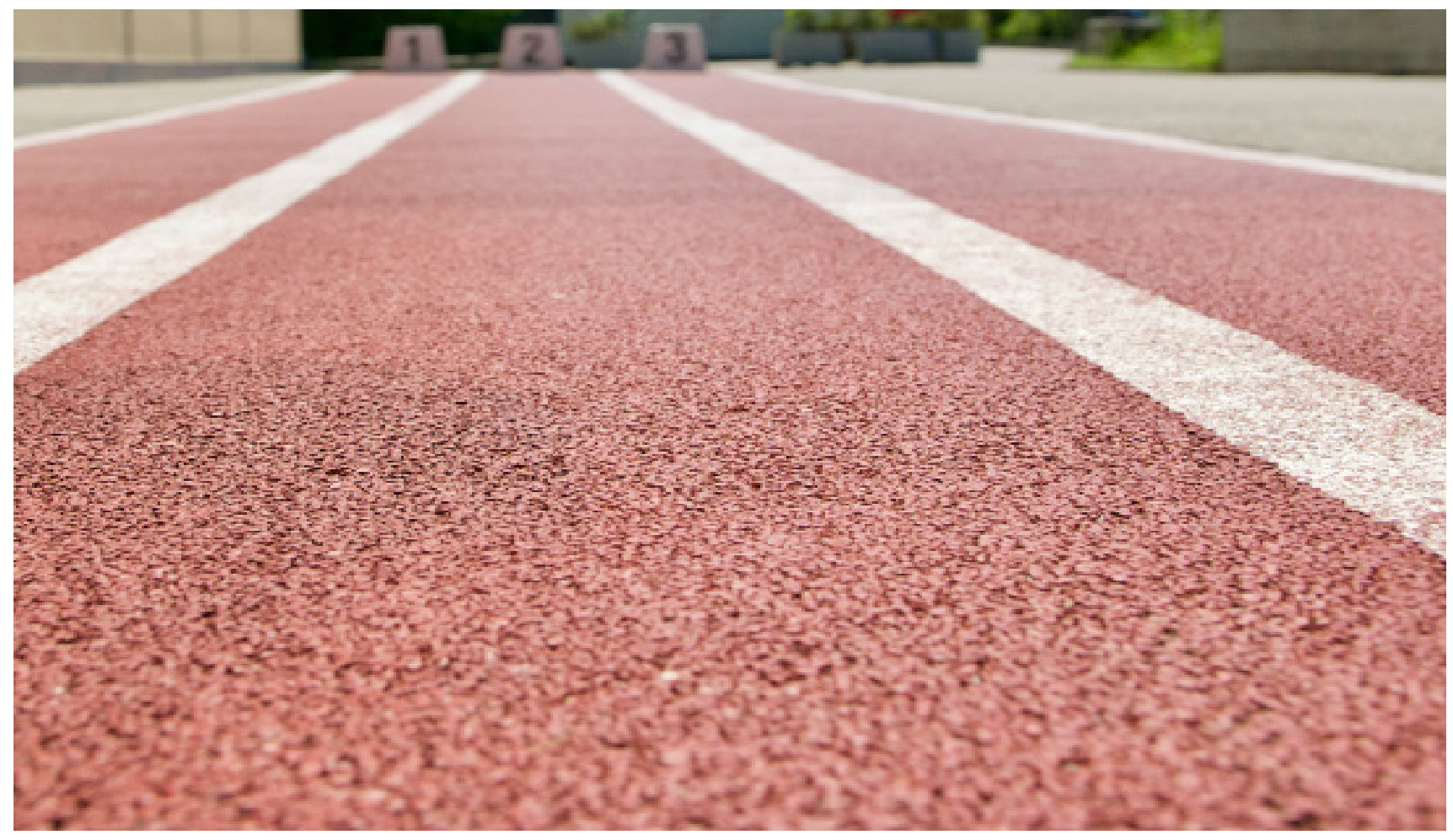

\title{
Soluciones innovadoras para problemas de cimentación sobre suelos cohesivos altamente plásticos
}

\section{Innovativesolutionsforfoundation problems on highly plastic cohesive soils}

Alexandra Castro-Sandoval

Ingeniero Civil, alexandracs@ufps.edu.co,

https://orcid.org/0000-0002-6522-7957, Universidad Francisco de

Paula Santander, Cúcuta, Colombia

Ciro Alfonso Melo-Pabón

Ingeniero Civil, ciroalfonsomp@ufps.edu.co,

https://orcid.org/0000-0001-8593-888X, Universidad Francisco de Paula

Santander, Cúcuta, Colombia

Gina Angulo-Blanquicetz

Ingeniero Civil, gina.angulo@utadeo.edu.co,

https://orcid.org/0000-0001-5020-1933,

Universidad Jorge Tadeo Lozano, Cartagena de Indias, Colombia 
Resumen

De manera general el objeto del presente estudio esidentificar las principales similitudes y diferencias entre métodos actuales de estabilización de suelos arcillosos con alta plasticidad, y determinar cuál de estos métodos es elmás eficientey permite mayores beneficios mecánicos en el ámbito del suelo como material de cimentación. Las propiedades del suelo que se analizan son: límite líquido (LL), límite plástico (LP), índice de plasticidad (IP), e índice de elastividad (IE), las cuales son comparadas a partir de los resultados de diferentes investigaciones para concluir cuál de los métodos permite que el suelo sea el mejor geo-material para cimentar. Se utilizan los suelos que son la muestra dentro de la población de suelos cohesivos son los que se clasifican según el sistema unificado $\mathrm{CH}$, que soy arcillas de alta plasticidad. Los resultados arrojaron que la estabilización realizada con polímeros arrojó los mejores resultados en cuanto a potencial de expansión, registrando valores bajos.

Palabras clave: Arcillas, estabilización, cenizas puzolánicas, cascarilla de arroz, polímeros

\section{Abstract}

In general terms, the purpose of this study is to identify the main similarities and differences between current methods of stabilizing clay soils with high plasticity, and to determine which of these methods is the most efficient and allows the greatest mechanical benefits in the area of soil as a foundation material. The soil properties that are analyzed are: liquid limit (LL), plastic limit (LP), plasticity index (IP), and elasticity index (IE), which are compared from the results of different investigations to conclude which of the methods allows the soil to be the best geomaterial for foundation. The soils that are the sample within the population of cohesive soils are those classified according to the unified system $\mathrm{CH}$, which are clays of high plasticity. The results showed that the stabilization made with polymers gave the best results in terms of expansion potential, registering low values.

Keywords: Clays, stabilization, pozzolanic ash, rice husk, polymers 


\section{6}

\section{Introducción}

El suelo sirve como estructura de soporte a las cimentaciones, están definidos como agregados no cementados de granos de minerales y con algún contenido de materia orgánica que se encontrara en estado de descomposición, y junto con ello se encontrara el líquido y el gas que ocupan los espacios vacíos entre las partículas sólidas [1].

De igual manera, Das en su libro destaca que Terzagui define al suelo como un agregado que se conforma naturalmente de partículas minerales que pueden desintegrarse por medios mecánicos de bajas magnitudes. Así mismo, se dice que es multifásico porque en él se encuentran las fases de estado sólido, líquido y gaseoso [2].

Además a lo largo del tiempo se han hecho diversos tipos de estudio sobre cómo mejorar las propiedades de los suelos arcillosos con alta plasticidad se ha podido determinar que esta tendencia fue influenciada desde la década de los años 50s [3] y que actualmente ha tomado gran relevancia, y se ha tenido tendencia a enfocarse a el uso de aditivos que beneficien el ambiente, el hecho de buscar nuevos materiales que permitan modificar las propiedades de los suelos sin que estos afecten de gran forma ecosistema permitiendo que se pueda reciclar, como es el caso del uso de residuos de construcción para estabilizar suelos o la de la utilización de la ceniza de cascara de arroz, cenizas puzolánicas como son las de origen volcánico o de desechos, o también puede hablarse del retal de vidrio molido y muchos otros ejemplos hay sobre esto es por ello que pareció una necesidad el poder comparar los nuevos descubrimientos a razón de que sean un aporte importante a este medio.

La cohesión como propiedad actuante en suelos cohesivos. "La cohesión se refiere a la atracción entre moléculas que se presenta en partículas" [4], siendo también está la característica distintiva, que permite decir que los geos materiales que poseen son las arcillas, según [5] este tipo de suelo es generalmente de las zonas cálidas y húmedas (tropicales) en donde normalmente la evo-traspiración supera la precipitación, sin embargo, puede suceder que se encuentren formaciones arcillosas en zonas frías o secas.

Del mismo modo, "un suelo que tenga el $20 \%$ de material arcilloso y que se encuentre en límites adecuados de humedad se comporta como un suelo cohesivo" [6]. Aunque hay inferencias en cuanto a este porcentaje de arcilla que debe estar presente en el suelo para ser clasificado como tal, pues según la NSR-10 en su título $\mathrm{h}$ dice que el suelo se considera cohesivo cuando pasa más del $30 \%$ de finos, si el LL>30\% y IP>10\%, esta clasificación se realiza según el sistema unificado.

Los suelos que son cohesivos y que poseen índices de plasticidad y expansión altos que se presentan como cambios de volumen significativos (expansión-contracción), dehecho son suelos que representan gran peligro para la infraestructura, pues estos cambios pueden significar que en ella se presenten problemas estructurales tan graves que implique el colapso, se hace necesaria caracterizar estos suelos desde su microestructura y esto hace referencia a la mineralogía que viene siendo, composición, interacciones con los fluidos del entorno y sus estados de esfuerzos si lo que se pretende es analizar el comportamiento mecánico [7].

Anisotropía como propiedad actuante del suelo cohesivo. Asimismo, cabe destacar que los suelos cohesivos son la manera más general de comenzar a describir el problema que se presenta en las cimentaciones puestas sobre ellos, ya que poseen características $y$ propiedades que pueden hacer que 
su comportamiento sea fatal si estas no son tenidas en cuenta antes y después de la construcción. Según [8] los suelos generalmente son isotrópicos y respecto a esto se estima la última capacidad de carga, sin embargo, los estratos de arcillas generalmente se depositan y consolidan en condiciones unidimensionales, y por lo general según [9] las naturales con frecuencia son anisotrópicas, característica brinda un agregado particular, pues ya el cálculo de la capacidad ultima de carga deberá realizarse con un ajuste [8].

Como se ha mostrado en el párrafo anterior, el hecho de la anisotropía tiene que ver en gran medida con los estados de esfuerzos en los suelos, [10] dice que dentro del margen de las propiedades de los suelos cohesivos expansivos tiene un valor sustancial pues estudia el comportamiento mecánico del suelo respecto a la dirección de la carga, de hecho, ella incide en las propiedades de rigidez, permeabilidad y resistencia. A lo largo del escrito se hará enfoque más que todo en la expansión y plasticidad ya que es la variable en donde más predomina la dificultad causada por los suelos cohesivos.

Composición química de los suelos cohesivos. Desde el punto de vista mineralógico, y de composición de las arcillas, según [4] se puede decir que son silicatos de aluminio y magnesio hidratados cuya estructura generalmente es cristalina y complicada. sus átomos están dispuestos en forma de láminas que pueden ser tetraedros de silicio u octaedros de aluminio, estas laminas forman tres grupos básicos de arcillas que son caolinitico, llitico y montmorilonitico por otro lado, [11] dice que los suelos que tengan contenidos altos de montmorilonitas o ilita son suelos expansivos y potencialmente peligrosos para la estructura de la edificación Asimismo, [12] dice que cuando un suelo tiene tan solo el $10 \%$ de minerales del tipo anterior mencionado le confiere instabilidad por expansión y contracción al adsorber agua.

Según [4] se clasifican en tres grupos básicos que son la caolinitico que proviene de la roca carbonatada orto-clástica y su estructura es una lámina silícica y otra lamina aluminica, las arcillas de este tipo son estables en presencia del agua, pues la unión de sus retículas no permite el paso de la molécula de agua, el segundo grupo es la montmorilonitico al cual pertenecen las betonias, que tiene una composición de dos laminas silícicas y una aluminica la unión entre las retículas es inestable por esa razón este tipo de arcillas sufren de fuertes expansiones, la ilitica tiene la estructura reticular similar a anterior grupo, esta se produce por la hidratación de las micas, una peculiaridad de ella es que tiende a formar grumos por presencia de iones de potasio lo que reduce un poco la probabilidad de ser expansivas, ya que las superficies no están tan expuestas al agua.

A demás [6] en el capítulo IV de que la plasticidad y las propiedades fisicoquímicas se relacionan de manera específica, ya que la primera sucede porque la película de agua que rodea la partícula de arcilla es más gruesa, y esto se produce debido al intercambio iónico entre la arcilla (monmolionita o ilita) y las moléculas de agua, esto sucede gracias a que las retículas son inestables.

Estabilización del suelo cohesivo. Las propiedades de la arcilla según [6] pueden cambiar al modificarse los cationes contenidos en sus complejos de adsorción, por esta razón a lo largo del tiempo el intercambio catiónico forzoso se usó para tratar suelos con intención de mejorar su comportamiento mecánico ese intercambio catiónico tiene mucho que ver con los enlaces químicos que presenta, pues el tipo de enlace que presenta la monmolionita y ilita permite el atraer moléculas y que se formen en enlaces entre ellas, que es el caso contrario 


\section{8}

de la caollita. Por esa razón el autor habla de que la estabilización consiste en cerrar esos enlaces, en no permitir que la molécula de agua se una a la de arcilla.

En síntesis, se hace necesario para que un suelo sea estable someterlo a cambios desde el punto de vista de la micro estructura a modificar la estructura química para de esta manera obtener cambios en las propiedades mecánicas, según [7] en la NSR10 [13] habla de las medidas que deben tomarse en suelos expansivos, dice que se pueden tratar de tres maneras que son: remplazamiento, tratamiento con cal o pre humedecimiento, por su puesto, el objeto del escrito es encontrar las técnicas de que hasta el momento existen que permitirán, actualizar o dar nuevos puntos de vista a los criterios del ingeniero, podría decirse que las que presenta la NSR 10 son las medidas tradicionales que se presentan a problemas de suelos cohesivos.

Problemas generados a la estructura en suelos cohesivos. Por otra parte, [12] dice que los problemas que generalmente se presentan en suelos cohesivos con altos índices de expansión son asentamientos diferenciales inducidos por la interacción suelo estructura - levantamientos producidos debido a los cambios volumétricos, estos problemas son posible solucionarlo interviniendo en la microestructura fisicoquímica, atreves de medios de estabilización. La expansividad depende de los límites de plasticidad, que son LC, LP, LL con ellos se llega a un valor de expansividad.

Soluciones innovadoras. De acuerdo a los análisis realizados de las diferentes fuentes, se van analizar cuatro tipos de formas innovadoras en las que se pretende dar solución a los suelos arcillosos. Los parámetros tenidos en cuenta para la elección de los datos fueron que los suelos sean clasificados según el sistema unificado como $\mathrm{CH}$, que se es una arcilla altamente plástica, pues generalmente este tipo de suelo es el que presenta mayor problema en las diferentes cimentaciones debido a su gran probabilidad de cambios de volumen por presencia.

Estabilización de suelos con ceniza puzolánica. Los autores [12] analizaron cómo cambian las propiedades de los suelos cuando se modifica la estructura química de ellos, pues dentro del estudio se usó ceniza puzolánica, dando un descripción breve de ellas estas están formadas por silicatos, óxidos de aluminio y cal libre, la última es la que da las propiedades puzolanas, el material se toma de la empresa termoeléctrica Termo-tasajero S.A. la cual se encarga de suplir la necesidad eléctrica en la región de Cúcuta, uno de los desechos de esta actividad son las cenizas, de las cuales se utilizan las que pasan el filtro electroestático.

Es un suelo de la formación León, en donde se tomaron 5 muestras de suelo, en la cual su característica inicial fue su índice de plasticidad (IP) era mayor que $30 \%$, su límite liquido era mayor a $50 \%$. A las diferentes muestras se les determino LL, LP, IP y LC cuando el volumen de ceniza de la muestra corresponde a $20 \%$, $25 \%$ y $30 \%$, la tabla 1 muestra los resultados obtenidos del ensayo.

Estabilización de suelos con ceniza de eco materiales.LosEco-materialeshacen referencia a productos que son desechos y que pudieran seraprovechados por para estas actividades sin que causen daños grades al ambiente, como son las cenizas de cascarilla de arroz y el bagazo de caña, y muchos otros materiales, en esta o portunidad se hará enfoque principalmente en la estabilización con ceniza de arroz. Este tipo de estabilización con ceniza de arroz resulta una alternativa útil, además de ser una solución a la alternativa de cuidar el medio ambiente ya que es un desecho agroindustrial que es poco aprovechado, siendo simplemente eliminado después de la quema. Según [14] en el año de producen 16mil Toneladas de ceniza de tamo 
de arro. Adicionalmente se define que la cascarilla de arroz está compuesta por una capa leñosa liviana con alto contenidos de sílice compuesta por un $50 \%$ de celulosa, $30 \%$ de lignina y 20 de sílice o Si O2, el material puede tener actividad puzolánica cuando está en presencia de $\mathrm{Ca}(\mathrm{OH}) 2$, compuesto que tiene presencia en la ceniza de la cascarilla, por lo que confirma que ella es un elemento útil en la mezcla para estabilizar suelos [15]. Se aplican porcentajes que corresponden al volumen de la muestra tomada de suelo en ceniza los cuales son $10 \%$, $20 \%$ y $30 \%$, el ensayo base que se utilizará en la tabla 1 y 2 , la cual permitirá comprar, son obtenidos de [5].

Estabilización de suelos por polímeros. Generalmente los polímeros actúan en el suelo formando una estructura impermeable al agua, [16]. De lo que puede deducirse que este material proporcionara resultados coherentes. El ejercicio principal es obtener cual es obtener mediante una serie de combinaciones el principal componente o porción que le corresponde polímero a la muestra de suelo para poder ser estbilizada, se ensayó entre mezclas de $4 \%$, $6 \%, 8 \%$ y $30 \%$. adicionalmente los resultados obtenidos se mostrarán en la comparación de la tabla 1 y 2.

Tabla 1. Comparación de los sistemas usados para la estabilización de suelo con cenizas cascarilla de arroz

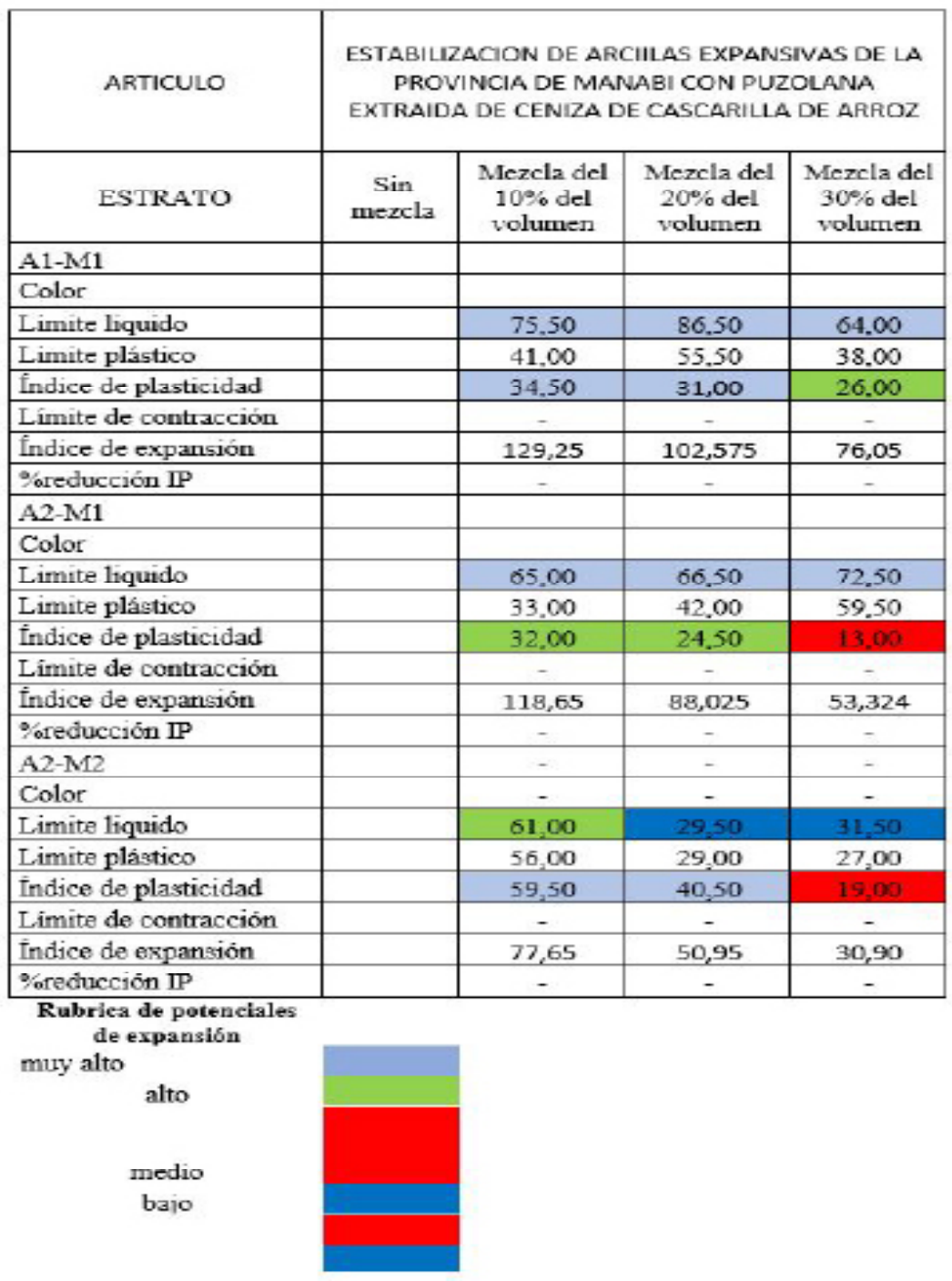


Tabla 2 Comparación de los sistemas usados para la estabilización de suelo con cenizas puzolánicas

\begin{tabular}{|c|c|c|c|c|}
\hline \multirow{2}{*}{$\begin{array}{l}\text { ARTICULO } \\
\text { ESTRATO }\end{array}$} & \multicolumn{4}{|c|}{ ESTABILIZACION DE SUELOS A TRAVES DE CENIZAS PUZOLANICAS } \\
\hline & Sin mezcla & $\begin{array}{c}\text { Mezcla del } 25 \% \text { del } \\
\text { volumen }\end{array}$ & $\begin{array}{c}\text { Mexcla del } 20 \% \text { del } \\
\text { volumen }\end{array}$ & Mezcla del $30 \%$ del volumer \\
\hline \multicolumn{5}{|l|}{ A1-M1 } \\
\hline Color & rojo & & & \\
\hline Limite liquida & 62,50 & 55,00 & 53,40 & 52,00 \\
\hline Limite pläatico & 23,39 & 21,46 & 21,75 & 26,39 \\
\hline Indice de plasticidad & 39,01 & 33.54 & 31,65 & 26,31 \\
\hline Limite de contracción & 12,66 & 14,46 & 13,87 & 15,09 \\
\hline İndice de expansićn & 1,63 & & & \\
\hline \%reducción IP & - & 14,02 & 18,87 & 32,56 \\
\hline \multicolumn{5}{|l|}{ A2-M1 } \\
\hline Color & marrón & & & \\
\hline Limite liquido & 64,40 & 49.00 & 48,002 & 43,10 \\
\hline Limite plástico & 24,16 & 17,60 & 17.85 & 24,16 \\
\hline Indioe de plasticidad & 40,24 & 31.40 & 30,15 & 23,94 \\
\hline Limite de contracción & 11,40 & 12,31 & 17,85 & 11,98 \\
\hline Indice de expansićn & 4,1 & & & \\
\hline Gireducción IP & - & 21.97 & 25,07 & 40,51 \\
\hline \multicolumn{5}{|l|}{$\mathrm{A} 2 \mathrm{-M} 2$} \\
\hline Color & verde-azul & & & \\
\hline Limite liquvido & 65.60 & 58,10 & 59,00 & 55,50 \\
\hline Limite plástico & 25,44 & 24,19 & 24,93 & 26,87 \\
\hline Indice de plazticidad & 40,16 & 33,91 & 34,07 & 28,63 \\
\hline Limite de contracción & 12,09 & 16,25 & 19,45 & 15,78 \\
\hline İodice de expansićn & 6,6 & & & \\
\hline Freducción IP & - & 15,56 & 15,16 & 28,71 \\
\hline \multicolumn{5}{|l|}{ A3-M1 } \\
\hline Color & rojo & & & \\
\hline Limite liquido & 67.50 & 54,70 & 58,00 & 54,40 \\
\hline Limite plástico & 22,45 & 21.81 & 24,37 & 22,10 \\
\hline Indice de plasticidad & 45,05 & 32,89 & 33,63 & 32,30 \\
\hline Limite de contracción & 12,55 & 20,17 & 16,68 & 18,29 \\
\hline İndice de expansićn & 1,04 & & & \\
\hline \%reducción IP & - & 26,99 & 25,35 & 28,30 \\
\hline \multicolumn{5}{|l|}{$\mathrm{A} 3-\mathrm{M} 2$} \\
\hline Color & verde-azul & & & \\
\hline Limite liquido & 59,70 & 60,60 & 51,70 & 52,20 \\
\hline Limite plástico & 21.97 & 24,06 & 22,41 & 23,39 \\
\hline Indioe de plesticidad & 37,73 & 36.54 & 29,29 & 28,81 \\
\hline Limite de centracción & 15,87 & 14,48 & 15,85 & 14,72 \\
\hline Indice de expansión & 1,63 & & & \\
\hline \%reducción IP & - & 3.15 & 22,37 & 23,64 \\
\hline
\end{tabular}

\section{Conclusiones}

Del análisis [12] en cuanto a la estabilización de suelos con ceniza puzolánica cabe destacar que según [13] se puede decir que la muestra A1-M1 con los resultados de mezcla en el $20 \%$ su límite liquido la clasifica como con potencial de expansión medio, en cuanto a límites de contracción se presenta en la tendencia a potenciales de expansión bajos, y en el índice de plasticidad, en la mayor parte las muestras presentaron en la mezcla de $30 \%$ de ceniza en el intervalo de 12-27 según [13] en índice plástico lo que indica que es un potencial de expansión medio, finalmente la muestra de suelo que mejor asimiló fue la A2-M1y el volumen de muestra fue $20 \%$ de ceniza, con un potencial de expansión medio. 
Respecto a el ensayo analizado [5] se pude establecer que según la [17] cuando la ceniza de cascarilla de arroz representa un $30 \%$ del volumen de la muestra es donde se obtienen óptimos resultados en cuanto al potencial de estabilización que es relativamente bajo. En cuanto a la mezcla analizada [16] corresponde el valor de potencial de expansibilidad baja en casi un $50 \%$ de el valor de la muestra sin alterar, y esto corresponde a la mezcla en donde el polímero era $8 \%$ del valor del volumen de la muestra.

Finalmente, se determinó que de las tres formas que se trataron dentro del documento, la estabilización realizada con polímeros arrojó los mejores resultados en cuanto a potencial de expansión, registrando valores bajos.

\section{Referencias}

[1] B.M. Das, Fundamentos de Ingeniería de cimentaciones, México: Cengage Learning, 2012.

[2] A.D. Rodríguez, Mecánica de suelos y propiedades de la naturaleza, México: Trillas, 2014.

[3] S.K. Buitrón Landeta, A.J Enríquez León, "Estudio de la estabilización de arcillas expansivas con ceniza del volcán Tungurahua". Escuela Politécnica Nacional, Quito, 2018.

[4] C. Crespo, Mecánica de suelos y cimentaciones, México: Limusa, 2004.

[5] E.A. Chicaiza Estévez, F.J. Oña Oña, "Estabilización de arcillas expansivas de la provincia de Manabí con puzolana extraída de ceniza de cascarilla de arroz" Escuela Politécnica Nacional, Quito, 2018.

[6] E.J. Badillo, Mecánica de suelos I, México: Limusa, 2005.
[7] L.J. Quintero y R.J. Gallardo, "Caracterización mineralógica de arcillas expansivas con fines de estabilización". Revista Ingenio, vol. 8, n. ${ }^{\circ}$, pp. 83-92, nov. 2015

[8] M.A. Al-Shamrani, A.A Baig Mogha, "Soluciones de forma cerrada para la capacidad de carga de zapatas en suelos anisotrópicos cohesivos", Revista ingenieria de construccion, vol. 30 no.2, 2015.http://dx.doi.org/10.4067/S071850732015000200003

[9] A.W. Bishop, "The strength of soils as engineering materials", Geotechnique, vol. 16, no. 2, pp. 91-130, June 1966

[10] J.A. Duque, "Experimentación y modelación constitutiva de arcillas anisotrópicas y su aplicación en monopiles", Universidad del Norte, Colombia, 2018

[11] J. Patrone y J.E. Prefumo, "La acción de los suelos expansivos sobre las cimentaciones; métodos de prevencion y control", Memoria de Trabajos de Difusión Científica y Técnica, $\mathrm{n}^{\circ}$. 4, pp. 51-74, 2005,

[12] C.H. Flórez-Góngora, Z.K. Caicedo-Osorio, R. Zárate-Caballero y B.A Contreras.Ortíz, "Estabilización química de suelos expansivos de San José de Cúcuta (Colombia) usando cenizas volantes" Respuestas, vol. 13, nº 2 , pp. 19-31, 2008.

[13] Asociación Colombiana de Ingeniería Sísmica, "Norma Colombiana de Construcción Sismo Resistente NSR-10 Título H - Estudios Geotécnicos.

[14] I. A. C. Murillo, "Mejoramiento del suelo blando de subrasante mediante la adición de cascarilla de arroz y su efecto en el módulo de resilente", Bogotá, 2016 


\section{2}

[15] M.B. Ramos y . D.F. Illigge Quintero, "Análisis de la modificación de un suelo altamente plastico con cascarilla de arroz y con ceniza volante para subrasante de un pavimento" Bogotá, 2017

[16] T. López-Lara, J.B. Hernández-Zaragozal, J. Horta-Rangel, A. Coronado-Márquez, V. M. Castaño-Meneses, "Polímeros para la estabilización volumétrica de arcillas expansivas", Revista Iberoamericana de Polímeros, vol. 11, no. 3, Mayo 2010 